\title{
Generative Adversarial Networks Enable Cross-Modality Super-Resolution in Fluorescence Microscopy
}

Hongda Wang ${ }^{1,2,3}$, Yair Rivenson ${ }^{1,2,3}$, Yiyin Jin ${ }^{1}$, Zhensong Wei ${ }^{1}$, Ronald Gao ${ }^{4}$, Harun Günaydın ${ }^{1}$, Laurent A. Bentolila ${ }^{3,5}$, Comert Kural ${ }^{6,7}$, Aydogan Ozcan ${ }^{1,2,3,8^{*}}$

${ }^{1 .}$ Electrical \& Computer Engineering Department, University of California, Los Angeles, Los Angeles, USA

2. Bioengineering Department, University of California, Los Angeles, Los Angeles, USA

3. California NanoSystems Institute, University of California, Los Angeles, Los Angeles, USA

4. Computer Science Department, University of California, Los Angeles, Los Angeles, USA

5. Department of Chemistry and Biochemistry, University of California, Los Angeles, Los Angeles, USA

6. Department of Physics, Ohio State University, Columbus, USA

7. Biophysics Graduate Program, Ohio State University, Columbus, USA

8. Department of Surgery, David Geffen School of Medicine, University of California, Los Angeles, Los Angeles, USA.

* Corresponding author: ozcan@ucla.edu

We present a generative adversarial network (GAN) [1] based framework that achieves cross-modality fluorescence image super-resolution [2]. This method takes a single low-resolution image as input and enhances its resolution to match the resolution of an image that is captured with a higher resolution microscopy setup without an iterative parameter search or the need for any prior knowledge on the point spread function or other imaging parameters.

The success of our method was demonstrated with wide-field fluorescence image transformation from low- to high-numerical aperture (NA) images, which revealed at the network output finer structures of microtubule and F-actin in bovine pulmonary artery endothelial cells (BPAEC) that were not resolved in the input images, as shown in Figure 1(a). In addition to higher spatial resolution, the deep neural network inferred images also demonstrate an extended depth-of-field compared to the ground truth images acquired with a higher NA objective lens [2].

We also applied this GAN-based super-resolution framework to transform confocal microscopy images to match the corresponding images of the same samples, acquired using a stimulated emission depletion (STED) microscope. We used $20 \mathrm{~nm}$ fluorescence particles to demonstrate the success of our approach: some of these nanoparticles were spaced within the classical diffraction limit and therefore were not resolved by the confocal microscope; however, they were successfully resolved by the deep neural network, matching the ground truth images acquired by a STED microscope. This cross-modality transformation framework was also verified by super-resolving the distribution of Histone3 sites within HeLa cell nuclei as shown in Figure 1(b). We emphasize that the excitation power of STED microscopy was three to ten-fold stronger than that of confocal microscopy and the depletion beam was orders of magnitude higher than the excitation beam.

We also applied our framework to super-resolve total internal reflection fluorescence (TIRF) microscopy images (Figure 1(c)), revealing the development and dissociation of clathrin-coated structures that can normally be resolved using structured illumination microscopy (SIM) based on a TIRF configuration [3] using 9 different structured illumination beams and exposures. Finally, we also 
demonstrated that a pre-trained network model can be generalized to new types of objects that were not introduced to the network in the training stage [2].

In summary, we believe that this GAN-based super-resolution framework can provide an avenue for the democratization of super-resolution microscopy, potentially leading to innovations and discovery in the fields of life sciences and biomedical imaging [4].

\section{References:}

[1] IJ Goodfellow et al., ArXiv14062661 Cs Stat (2014).

[2] H Wang et al., Nature Methods 16 (2019), p. 103.

[3] D Li et al., Science 349 (2015), p. aab3500.

[4] HW and YR are equal contributing authors.
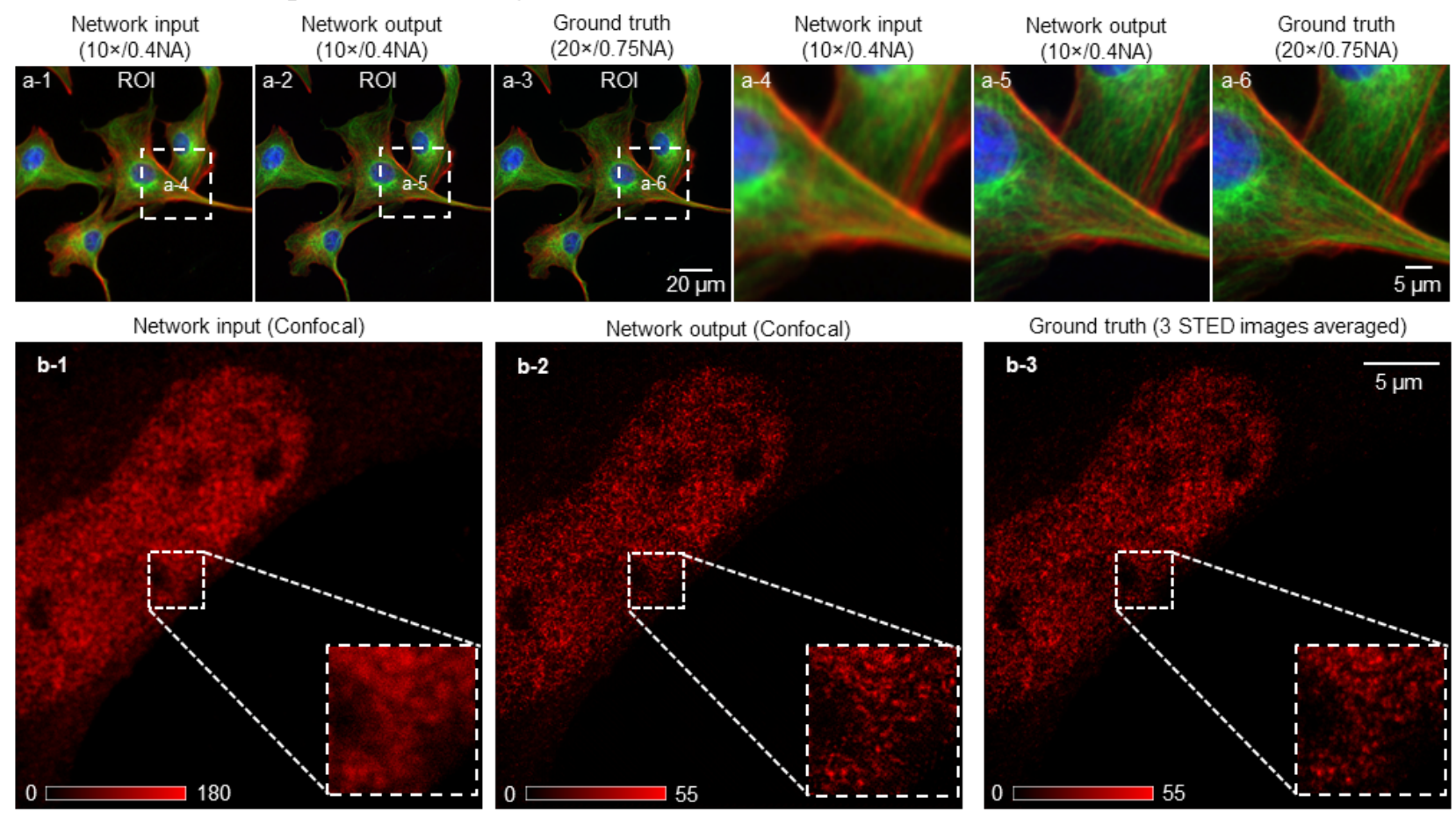

Ground truth (3 STED images averaged)
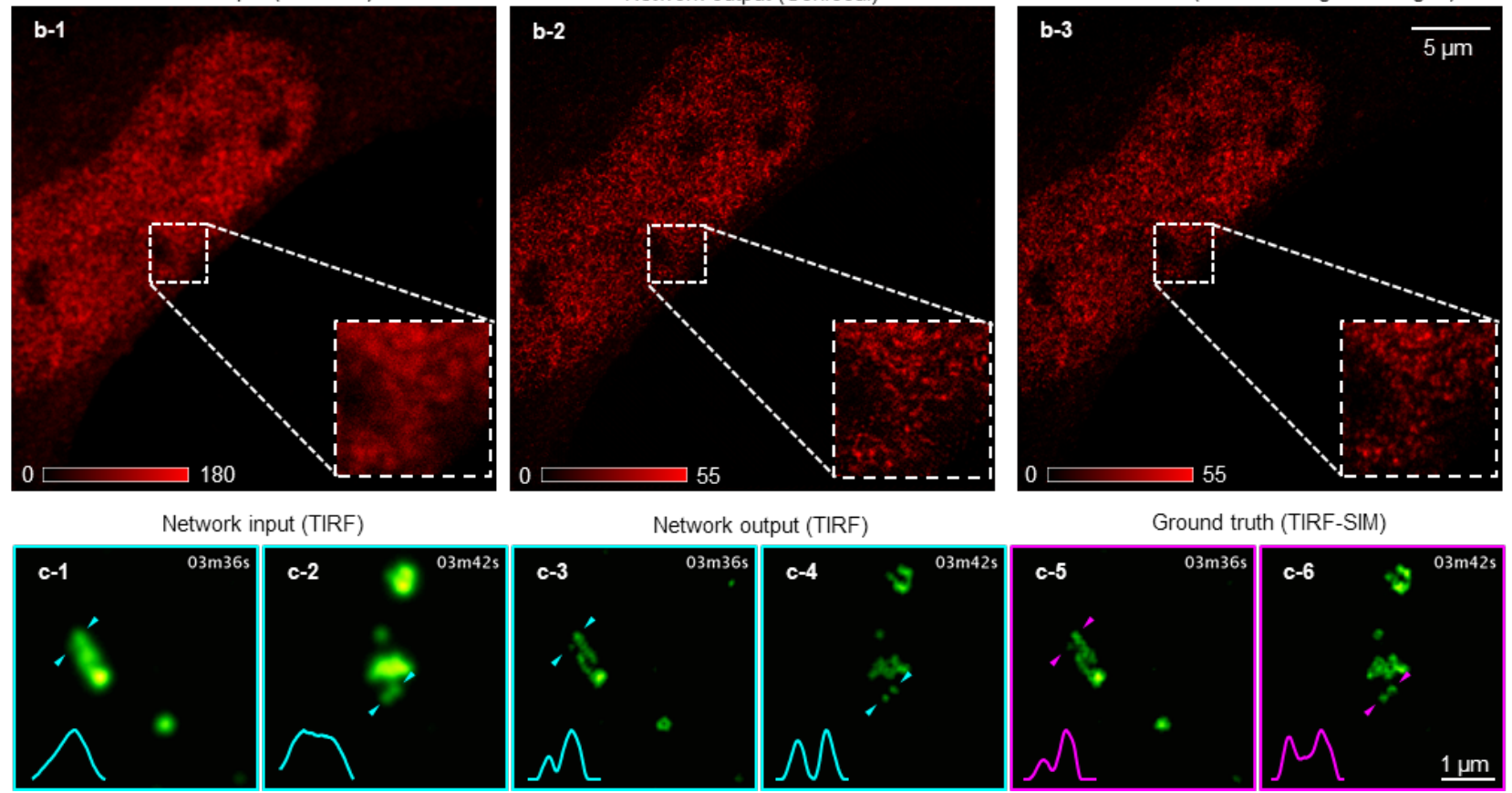

Ground truth (TIRF-SIM)

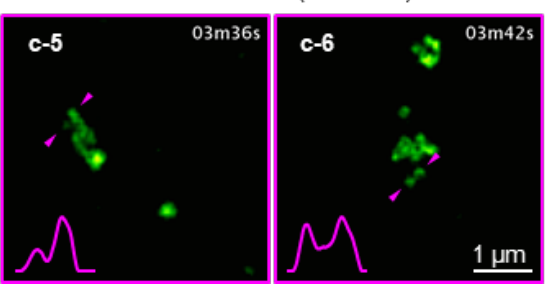

Figure 1. Cross-modality super-resolution in fluorescence microscopy enabled by deep learning. (a) Resolution enhancement in wide-field fluorescence microscopy. (b) Super-resolution image transformation of a confocal microscope image reveals the distribution of Histone 3 sites within HeLa cell nuclei and matches the resolution of STED images of the same samples. (c) Super-resolution image transformation of TIRF images resolves finer structures of clathrin-coated structures, matching the resolution of TIRF-SIM images of the same samples. 